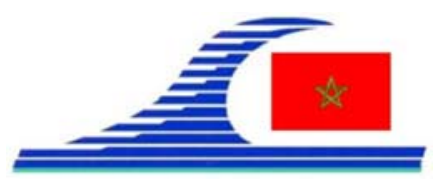

Conférence Méditerranéenne Côtière et Maritime

EDITION 2, TANGER, MAROC (2011)

Coastal and Maritime Mediterranean Conference

Disponible en ligne - http://www.paralia.fr - Available online

\title{
Les hydrocarbures non aromatiques dans les eaux de la Méditerranée marocaine Ouest (Kabila-Kaâ Srass)
}

\author{
Saida BOUZID ${ }^{1}$, Soumaya KHANNOUS ${ }^{1}$, Ahmed RAISSOUNI ${ }^{1}$, \\ Karim EL ARRIM ${ }^{1}$, Ioanna BOULOUBASSI ${ }^{2}$, \\ Alain SALIOT ${ }^{2}$, Hassan ER-RAIOUI ${ }^{1}$
}

1. Université Abdelmalek Essaâdi, Ancienne route de l'aviation, BP. 416, Tanger, Maroc. sur24@yahoo.fr

2. Université Pierre et Marie Curie, 4 Place Jussieu, 75252 Paris, , France.

\section{Résumé :}

Les concentrations des hydrocarbures non aromatiques ont été déterminées au niveau des eaux de surface (matière particulaire) de la Méditerranée Marocaine Ouest (Frange littorale Kabila-Kaâ Srass) afin de déterminer l'état de la qualité des eaux de baignade vis-à-vis de la contamination par les hydrocarbures. Ainsi, les hydrocarbures totaux ont été extraits et fractionnés sur colonnes de silice en hydrocarbures aromatiques et hydrocarbures non aromatiques. Cette dernière fraction analysée par des techniques chromatographiques, montre des concentrations importantes (HNA: 0,78-68,13 $\mu \mathrm{g} / \mathrm{l}$ ) comparativement à d'autres endroits en Méditerranée.L'étude qualitative basée sur l'analyse des différents indicateurs géochimiques (n-alcanes, hopanes, isoprénoides, UCM, etc.) a permis de mettre l'accent sur la nature biogénique des apports, principalement continentale avec une faible présence de la trace des apports pètrogèniques.

\section{Mots-clés :}

Hydrocarbures non aromatiques - Matière particulaire - Marqueurs géochimiques Frange littorale Kabila-Kaâ Srass - Méditerranée marocaine Ouest

\section{Introduction}

La façade méditerranéenne marocaine n'est pas aussi importante que celle de l'Atlantique, mais elle assure un rendement économique élevé basé sur les activités touristiques et de pêche sur le tourisme et la pêche. De par son positionnement, ce littoral méditerranéen marocain est exposé à toute sorte de pollution et en particulier à la présence des hydrocarbures.

La frange littorale de Kabila-Kaâ Srass, qui fait partie de la Méditerranée marocaine, est une région soumise aux risques naturels. Mis à part l'activité du tourisme, c'est une zone caractérisée par l'installation des ports de pêche et de plaisance qui subit d'une part, l'influence des évacuations urbaines et industrielles de la ville de Tétouan et des 
La connaissance de la Mer :

un vecteur du développement durable en Méditerranée

agglomérations de M'diq, Martil et Oued Laou, et d'autre part, celle des activités de navigation et de transport de passagers, de marchandises et d'hydrocarbures.

Cette propension d'effluents industriels et d'hydrocarbures pose problème et constitue une menace pour la qualité des eaux des côtes marocaines. Pour répondre à ce problème, une étude sur l'état de la pollution par les hydrocarbures non aromatiques a été menée (HNA) au niveau de la fraction particulaire des eaux de baignade au moyen de l'analyse des marqueurs géochimiques : n-alcanes et isoprénoïdes ( $\mathrm{Pr}$ : Pristane, $\mathrm{Ph}$ : Phytane).

\section{Matériels et méthodes}

Cinq sites ont été choisis pour l'échantillonnage en tenant compte de la situation d'arrivée des rejets urbains et de la proximité aux ports (figure 1). La campagne d’échantillonnage a été réalisée durant la saison pluviale de 2004.

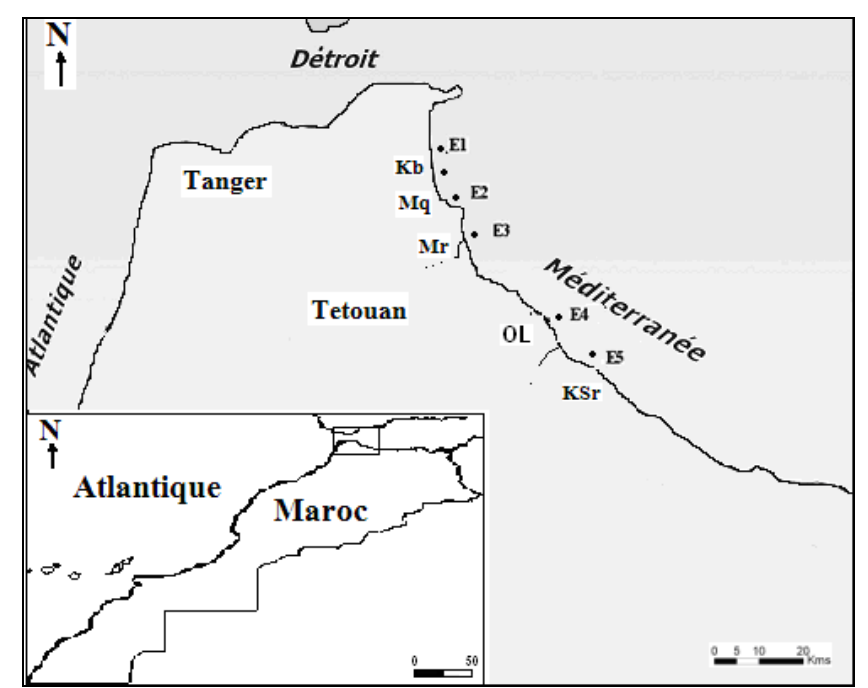

Figure 1. Localisation des points de prélèvement d'eau au niveau de la frange littorale Kabila-Kaâ Srass

(Kb : Kabila ; Mq : M’diq ; Mr : Martil ; OL : Oued Laou ; KSr : Kaâ Srass).

Par station, $5 \mathrm{l}$ d'eau ont été prélevés et filtrés sur un filtre de verre Whatman GF/F (0.7 $\mu \mathrm{m}$ de pore et $142 \mathrm{~mm}$ de diamètre). Les filtres contenant les particules sont ensuite congelés jusqu'au traitement ultérieur en laboratoire.

L'extraction a été réalisée selon le protocole de BLIGH et DYER (1959) modifié, où le chloroforme a été remplacé par le dichlrométhane. Les échantillons, dopés par des étalons internes (C24D50), ont été extraits 2 fois au sonicateur avec un mélange d'eau saline $S$ (7.3\%), dichlorométhane, méthanol $(2: 1: 0.8, \mathrm{v} / \mathrm{v} / \mathrm{v})$ et filtrés. Le filtrat est ensuite extrait au dichlorométhane, concentré, séché sur MgSO4 pendant 24 h, filtré sur verre fritté et concentré à nouveau jusqu’à $2 \mathrm{ml}$. La séparation a été réalisée sur colonne 
de gel de silice en deux fractions: F1 et F2 représentant les aliphatiques et les aromatiques successivement par l'hexane et le mélange hexane/toluène (9:1).La fraction F1 a été analysée sur GC/FID et GC/MS. Le chromatographe utilisé est un HP Agilent muni d'un détecteur à ionisation de flamme FID, d'une précolonne pour l'injecteur et d'une colonne CP-sil 5CB50 m avec l'hélium comme gaz vecteur. Le programme utilisé pour cette analyse est le suivant : la température du four est de $60^{\circ} \mathrm{C}$ pour une minute et la vitesse de chauffe est de $25^{\circ} \mathrm{C} /$ min jusqu'à $100^{\circ} \mathrm{C}$ puis de $3^{\circ} \mathrm{C} / \mathrm{min}$ jusqu'à $360^{\circ} \mathrm{C}$, avec un isotherme à $310^{\circ} \mathrm{C}$ pour $100 \mathrm{~min}$.

La fraction F1 a également fait l'objet d'une analyse sur GC/MS au chromatographe HP Agilent/MS/Précolonne muni d'une colonne DB5 50m avec l'hélium comme gaz vecteur. Les conditions d'analyses sont identiques à celles décrites pour la GC/FID.

\section{Résultats et interprétation}

L'analyse de la fraction des hydrocarbures non aromatiques révèle des concentrations qui oscillent entre 20,28 et 68,13 $\mu \mathrm{g} / \mathrm{l}$ (tableau 1).

Tableau 1. Concentrations et données chromatographiques relatives aux HNA des échantillons d'eau prélevés au cours de la période pluviale de la frange Kabila-Kaâ Srass (HNA et T-alk sont représentées en $\mu \mathrm{g} / \mathrm{l}$ ).

\begin{tabular}{lllllllll}
\hline & HNA & T-n-alk & $\mathbf{P r} / \boldsymbol{P h}$ & $\mathbf{C 1 7 / P r}$ & $\mathbf{C 1 8 / P h}$ & $\mathbf{C P I}$ & \%C>25 & C29/C17 \\
\hline E1 & 20,28 & 0,16 & 26,88 & 1,37 & 33,31 & 1,15 & 22,61 & 0,72 \\
E2 & 31,09 & 0,11 & 0,73 & 1,83 & 1,18 & 1,75 & 23,77 & 0,54 \\
E3 & 42,13 & 3,71 & 1,33 & 2,33 & 1,98 & 1,02 & 89,13 & 12,6 \\
E4 & 68,13 & 0,37 & 0,76 & 1,94 & 2,01 & 1,07 & 56,79 & 6,58 \\
E5 & 22,97 & 0,13 & 0,65 & 2,12 & 3,28 & 1,84 & 19,91 & 2,07 \\
\hline
\end{tabular}

Les valeurs les plus importantes sont enregistrées au niveau des points E3 et E4 qui se situent respectivement à proximité des embouchures de l'Oued Martil et de l'Oued Laou. Les chromatogrammes des HNA montrent une distribution du n-C14 au n-C37 ainsi que l'absence des composés non résolus UCM généralement attribués à des produits issus de la dégradation des hydrocarbures (figure 2) (ZAGHDEN et al., 2005). La distribution des n-alcanes est dominée par les composés de faible poids moléculaire pour tous les échantillons (tableau 1), à l'exception des sites de Oued Martil et de Oued Laou où le pourcentage des composés à nombre de carbone inférieure à 25 est supérieur à 50\%. Ces derniers montrent des figures de distribution régulière unimodale à Martil et bimodale à Oued Laou, centrée autour des composés n-C20 et n-C29 reliés respectivement à une origine bactérienne (GRIMALT et al., 1988) et à une origine continentale (plantes supérieures). Le n-C15 et le n-C17, généralement liés à la trace phytoplanctonique, sont présents en tant que composés mineurs. Pour les autres sites, la 
La connaissance de la Mer :

un vecteur du développement durable en Méditerranée

distribution est irrégulière et montre la présence des différentes composantes naturelles marine et continentale. Les différents rapports calculés ( $\mathrm{Pr} / \mathrm{Ph}, \mathrm{n}-\mathrm{C} 17 / \mathrm{Pr}, \mathrm{n}-\mathrm{C} 18 / \mathrm{Ph}$ ) indiquent un apport biogénique de la matière organique excepté pour le rapport $\mathrm{Pr} / \mathrm{Ph}<1$ qui suggère un apport anthropique excepté pour Martil et Kabila. D’autre part, la trace pétrogénique est indiquée par les valeurs d'indice d'imparité (CPI) proche de l'unité (ZAGHDEN et al., 2005).

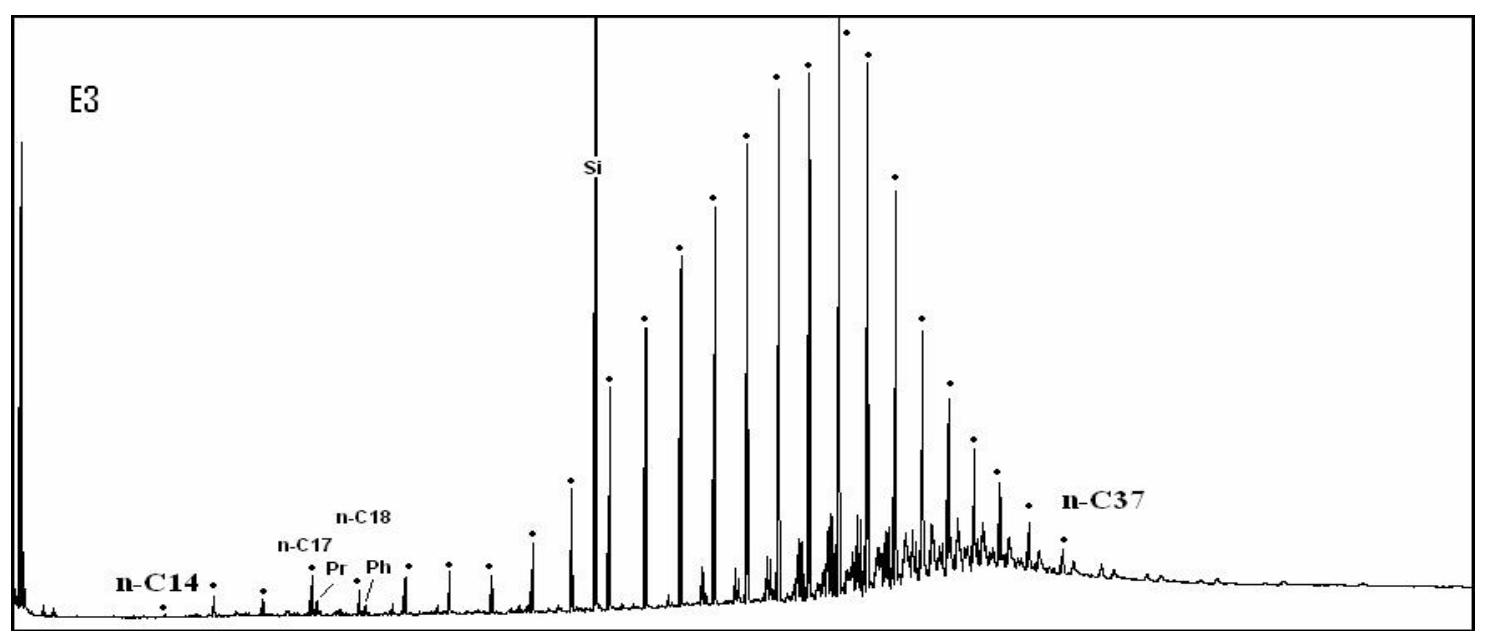

Figure 2. Chromatogramme de la fraction des hydrocarbures non aromatiques obtenu par GC/FID pour l' échantillon d'eau (E3) prélevé de la frange littorale Kabila-Kaâ Srass.

\section{Conclusion}

L'analyse de la fraction particulaire en provenance de la région Kabila-Kaâ Srass a mis en évidence l'apport important des hydrocarbures d'origine biologique et plus précisément un apport continental provenant des plantes supérieures. L'origine marine a été également démontrée. Néanmoins, les résultats obtenus ne permettent pas de conclure sur la présence marquée d'une contamination pétrolière bien que les indices d'indication d'origine et d'évolution militent en faveur d'une telle contribution.

\section{Références bibliographiques}

BLIGH E.G, DYER W.J. (1959). A rapid method of total lipid extraction and purification. Canadian journal of biochemistry and Physiology, ${ }^{\circ}$ 37, pp 911-917.

GRIMALT J.O., TORRAS E., ALBAIGES J. (1988). Bacterial reworking of sedimentary lipids during sample storage. Advances in organic geochemistry. Organic Geochemistry. $\mathrm{n}^{\circ}$ 13, pp 741-746.

ZAGHDEN H., LOUATI A., KALLEL M., ELLEUCH B., SALIOT A., OUDOT J. (2005). Hydrocarbons in surface sediments from the Sfax coastal zone, (Tunisia) Mediterranean Sea. Marine Pollution Bulletin, n 50, pp 1287-1294. 